\title{
DistribuiçÃo nOS Compartimentos Ambientais dOS HeRbicidas Utillizados nas Culturas de Algodão, Café e Citros ${ }^{1}$
}

\author{
Distribution of Environmental Compartments of Herbicides Used in the Cotton, Coffee and \\ Citrus Cultures
}

PLESE, L.P.M. ${ }^{2}$, SILVA, C.L. ${ }^{3}$ e FOLONI, L.L. ${ }^{4}$

\begin{abstract}
RESUMO - O objetivo deste trabalho foi avaliar o destino ambiental dos herbicidas acetochlor, 2,4-D, diuron, clomazone, thidiazuron, paraquat, simazine, fluazifop-p-butil, clethodim, oxyfluorfen, flumioxazin, carfentrazone-ethyl, ametrina, trifluralin e MSMA em áreas de cultivo de algodão, café e citros, utilizando o modelo de fugacidade nivel I. Na metodologia foram utilizadas basicamente as características físico-químicas dos herbicidas, os compartimentos ambientais e as equações de fugacidade. A avaliação preliminar do risco de contaminação pelo uso de herbicidas nas culturas de algodão, café e citros pode ser feita de forma expedita a partir das propriedades fisico-químicas desses produtos, aplicando o modelo de fugacidade nivel I. Para a maioria dos herbicidas avaliados, o compartimento água foi o mais vulnerável. O estudo de avaliação da predição em que se empregou o nivel de fugacidade I demonstrou ser uma ferramenta importante no destino ambiental dos herbicidas estudados para as culturas de algodão, café e citros.
\end{abstract}

Palavras-chave: comportamento, destino ambiental, fugacidade, nível I, herbicidas.

ABSTRACT - The aim of this paper was to evaluate the environmental fate of herbicides (acetochlor, 2,4-D, diuron, clomazone, thidiazuron, paraquat, simazine, fluazifo-p-butil, clethodim, oxufluorfen, flumioxazin, carfentrazone-ethyl, ametrina, trifluralin and MSMA) in cotton, coffee and citrus cultivation areas, applying the level I fugacity model. The methodology basically used the chemical and physical characteristics of the pesticides, environmental compartments and the fugacity equations. The preliminary evaluation of risk of contamination due to the use of these pesticides on the cultures studied was carried out swiftly, based on the chemical and physical properties of these products as the level I fugacity model was applied. For most of the herbicides evaluated, the water compartment was the most vulnerable. The prediction evaluation study using fugacity level I was found to be a relevant tool for the environmental fate of the herbicides studied for cotton, coffee and citrus cultures.

Keywords: behavior, environmental fate, fugacity, level I, herbicides.

\section{INTRODUÇÃO}

Os herbicidas foram desenvolvidos para controlar as plantas daninhas e são aplicados em pré-plantio, pré-emergência ou pós-emergência, dependendo das condições da área. Os principais herbicidas aplicados na cultura do algodão são 2,4-D, flumioxazin, paraquat, diuron, carfentrazone-ethyl, trifluralin, clomazone, clethodim, fluazifop-p-butil, flumioxazin, MSMA, diuron e paraquat; para o café, paraquat, diuron e 2,4-D, oxyfluorfen, acetochlor, ametrina e simazine; e para citros, paraquat, diuron, 2,4-D, thidiazuron, oxyfluorfen e flumioxazin. Esses herbicidas apresentam diferentes propriedades físico-químicas e, quando aplicados na

1 Recebido para publicação 24.9.2007 e na forma revisada 18.2.2009.

2 Bolsista DCR do CNPq/FUNTAC, vinculado ao Centro de Ciências Biológicas da Natureza da Universidade Federal do Acre, BR 364, km 04, Distrito Industrial, Rio Branco-Acre, <lpmplese@yahoo.com>; ${ }^{3}$ Aluno de Doutorado do Curso de Pós-Graduação da Faculdade de Engenharia Agrícola da Universidade Estadual de Campinas; ${ }^{4}$ Professor Col., Dr., do Curso de Pós-Graduação da Faculdade de Engenharia Agrícola da Universidade Estadual de Campinas, <lfoloni@gmail.com>. 
planta ou no solo, tendem a caminhar para os compartimentos de maior afinidade, ou seja, os mais vulneráveis. Para estudos de avaliação de impacto ambiental ou risco ambiental, é necessário o desenvolvimento de experimentos de campo ou mesmo em condições de laboratório para entender o comportamento e/ ou o destino ambiental das moléculas. No Brasil ainda é pequeno o número de trabalhos que tratam desse tema, e a maioria das informações é do exterior, nem sempre representando as condições brasileiras.

Além disso, as análises de resíduos de herbicidas são muito onerosas. Para evitar gastos desnecessários com análise de resíduos de compartimentos não-vulneráveis e também prever onde o herbicida tende a se concentrar, antes da sua aplicação, iniciou-se o desenvolvimento de modelos matemáticos dos mais diferentes niveis de complexidade para reproduzir o seu destino.

Muitos países, comos Estados Unidos, já vêm utilizando modelo matemático para novos registros de produtos que apresentem características de risco ambiental, além de produtos que necessitam de novos estudos para receber o registro novamente.

Uma forma possivel de predição dos destinos consiste na utilização de modelos matemáticos, que, com uma série de dados e modelos ajustados, procuram determinar como quais compartimentos podem ser atingidos. Os bancos de dados fornecem maior número de informações para elaboração de modelos matemáticos que podem servir de ferramenta para posterior experiência de campo.

Embora o conceito de fugacidade não seja novo, no Brasil apenas alguns estudos teóricos e raras pesquisas de campo avaliam essa propriedade, principalmente para o comportamento de produtos fitossanitários no meio ambiente. A fugacidade pode ser um novo caminho e, talvez, o melhor meio de quantificar o transporte, a bioacumulação e a transferência entre compartimentos (ar, água, solo, sedimento, biota, sólido suspenso e outros).

Presentemente, encontram-se na literatura modelos matemáticos de niveis dos mais simples até os mais complexos, em que são utilizadas informações como tipo de solo, temperatura, umidade, característica físico-química da molécula etc. Os estudos com modelos matemáticos no Brasil são recentes para avaliar o destino ambiental dos herbicidas.

A fugacidade é um conceito da termodinâmica que explica os desvios de comportamento dos gases reais com respeito aos gases ideais no estudo de energia livre em um processo de expansão, isotérmico, reversivel e infinitesimal. Utilizando-se desse conceito, iniciou-se aplicação para entender o destino ambiental dos herbicidas no ambiente por meio de modelo matemático.

O modelo de fugacidade é dividido em quatro niveis de complexidade - denominados de I, II, III e IV - e aplicado em sistema ambiental hipotético (Mackay, 1991), constituído dos compartimentos ar, água, solo, sedimento sólido suspenso e biota.

Os parâmetros físico-químicos e ambientais no modelo de fugacidade nível I (Mackay, 1991; Paraíba, 1999) permitem estimar valores teóricos de concentrações ambientais esperadas de cada um dos herbicidas utilizados em um determinado sistema de produção agrícola, aplicando fórmulas matemáticas (Mackay \& Paterson, 1981; Mackay, 1991) para exprimir o grau de letalidade dessas concentrações em cada um dos compartimentos ambientais considerados na modelização.

O nível I calcula a distribuição do pesticida entre os compartimentos, assumindo equilíbrio termodinâmico dos coeficientes de partição em estado de equilíbrio estacionário. Para o modelo de fugacidade nível II, há adição da advecção e degradação da substância nos compartimentos (Mackay, 1991). O nivel III de fugacidade supõe que as fugacidades estão em estado estacionário não equilibrado, podendo ter diferentes valores, os quais são determinados por um sistema linear de equações algébricas, representando um sistema ambiental compartimental, onde há advecções, degradações, emissões e transferência de substância entre os compartimentos (Mackay, 1991). Por último, tem-se o nivel IV de fugacidade, o qual é descrito por um sistema linear de equações diferenciais ordinárias (Mackay, 1991) ou por sistema dinâmico de controle (Bru et al., 1998; Paraiba et al., 1999).

No Brasil, estudos de herbicidas em que se utiliza o modelo de fugacidade são recentes 
e também há pouquíssimas informações de estudos de campo sobre o comportamento e destino ambiental.

Em trabalhos realizados com pesticidas (clomazone e 2,4-D) em que se aplicou o modelo de fugacidade nivel I, Plese et al. (2006a) e Silva et al. (2007) verificaram a preferência pelo compartimento água. Enquanto o herbicida acetochlor apresentou tendência a se distribuir para água, solo, caule e raiz, e o paraquat demonstrou ter preferência pelos compartimentos solo e sedimento (Plese et al., 2006a, b).

Para o nivel II de fugacidade, Yenigün \& Sohtorik (1995) utilizaram vários inseticidas organofosforados e verificaram que a maioria dos produtos estudados tendeu a se distribuir pelos compartimentos água e solo.

Quanto ao nível IV, Paraíba et al. (2007) conduziram um experimento de campo com arroz irrigado e observaram a distribuição do inseticida carbofuran pelos compartimentos água, solo, planta e ar em ordem decrescente.

O objetivo do presente trabalho foi avaliar o destino ambiental dos herbicidas acetochlor, 2,4-D, diuron, clomazone, thidiazuron, paraquat, simazine, fluazifop-p-butil, clethodim, oxyfluorfen, flumioxazin, carfentrazone-ethyl, ametrina, trifluralin e MSMA em áreas de cultivo de algodão, café e citros, utilizando o modelo de fugacidade nível I.

\section{MATERIAL E MÉTODOS}

O método da avaliação descreve as propriedades físico-químicas dos principais herbicidas usados nas culturas de algodão, café e citros (Tabela 1). Os dados dos herbicidas necessários para calcular o seu potencial de distribuição unidade ambiental padrão através do modelo de fugacidade nível I são: o estado iônico, a constante de dissociação ácida $(p K a)$ ou básica $(p K b)$, a massa molar $(M)$, a temperatura de fusão $\left(P_{f}\right)$, a pressão de vapor $\left(P_{\mathrm{v}}\right)$, a solubilidade aquosa $(S)$, o coeficiente de partição entre octanol e a água $\left(K_{\text {ou }}\right)$, o coeficiente de partição entre o carbono orgânico e a água $\left(K_{\circ c}\right)$ e o fator de bioconcentração $(B C F)$ (Tabela 2) (Tomlin, 2001).

O modelo de fugacidade nível I foi descrito de tal forma que a fugacidade se relaciona com a concentração $C$, em $\mathrm{mol} \mathrm{m}^{-3}$, através da capacidade de fugacidade $Z$, dada em $\mathrm{mol} \mathrm{m}^{-3} \mathrm{~Pa}^{-1}$. Assim, pode-se calcular a concentração de um composto em um compartimento pela equação $C=Z f(7)$, em que $f$ é a fugacidade dada em unidades de Pascal Pa (Mackay \& Paterson, 1981; Mackay, 1991).

Em estado de equifugacidades, os compartimentos com alta capacidade de fugacidade terão altas concentrações do composto. Portanto, a capacidade de fugacidade é uma medida da 'solubilidade' do composto em um determinado compartimento. Dessa forma, cada compartimento necessita que seja definida a

Tabela 1 - Principais herbicidas e sua época de aplicação em áreas de algodão, café e citros

\begin{tabular}{|c|c|c|c|}
\hline Época de aplicação & Algodão & Café & Citros \\
\hline Pós-Plantio & \multicolumn{2}{|c|}{} & $\begin{array}{c}\text { Paraquat, Diuron, } \\
\text { 2,4-D, Oxyfluorfen e } \\
\text { Flumioxazin }\end{array}$ \\
\hline Manejo & $\begin{array}{c}\text { 2,4-D, Flumioxazin, } \\
\text { Paraquat, Diuron, } \\
\text { Carfentrazone-ethyl e } \\
\text { Thidiazuron }\end{array}$ & Paraquat, Diuron e 2,4-D & \\
\hline Pré-Emergência & $\begin{array}{c}\text { Trifluralin, Clomazone e } \\
\text { Diuron }\end{array}$ & $\begin{array}{c}\text { Oxyfluorfen, Acetochlor, } \\
\text { Ametrina e Simazine }\end{array}$ & \\
\hline Pós-Emergência & Cletodim, & & \\
Estádios 1 a 6 & Fluazifop-p-butil, & & \\
& Flumioxazin, & & \\
& MSMA, Diuron e & & \\
\end{tabular}


Tabela 2 - Propriedades físico-químicas dos principais herbicidas utilizados em área de cultivo de algodão, café e citros

\begin{tabular}{|l|c|c|c|c|c|c|c|}
\hline \multirow{2}{*}{ Herbicida } & \multicolumn{9}{|c|}{ Propriedade fisico-química } \\
\cline { 2 - 8 } & $\begin{array}{c}\mathrm{PM} \\
\left(\mathrm{g} \mathrm{mol} \mathrm{I}^{-1}\right)\end{array}$ & $\begin{array}{c}\mathrm{PV} \\
(\mathrm{mPa})\end{array}$ & $\begin{array}{c}\text { Sw } \\
\left(\mathrm{mg} \mathrm{L}^{-1}\right)\end{array}$ & Koc & logKow & $\begin{array}{c}\mathrm{H} \\
\left(\mathrm{Pa} \mathrm{m} \mathrm{mol}^{-1}\right)\end{array}$ & $\begin{array}{c}\mathrm{pKa} \text { ou } \\
\mathrm{pKb}\end{array}$ \\
\hline Acetocholor & $269,8^{*}$ & $265^{*}$ & $223^{*}$ & $1,02^{* *}$ & $4,14^{*}$ & $3,83 \times 10^{-1^{*}}$ & - \\
\hline Ametrina & $227,3^{*}$ & $0,365^{*}$ & $200^{*}$ & $300^{*}$ & $2,63^{*}$ & $4,1 \times 10^{-4^{*}}$ & $4,1^{*}$ \\
\hline Carfentrazone & $412,2^{*}$ & $1,6 \times 10^{-2^{*}}$ & $12^{*}$ & $25^{*}$ & $3,36^{*}$ & $2,47 \times 10^{-4^{*}}$ & - \\
\hline Clethodim & $359,9^{*}$ & $<1 \times 10^{-2^{*}}$ & $5.520^{* *}$ & $0,12^{* *}$ & $2,39^{* *}$ & $4,56 \times 10^{-3 * *}$ & - \\
\hline Clomazone & $239,7^{*}$ & $19,2^{*}$ & $0,001^{*}$ & $356^{*}$ & $2,5^{*}$ & $4,19 \times 10^{-3^{*}}$ & - \\
\hline 2,4-D & $221^{*}$ & $1,86 \times 10^{-2^{*}}$ & $20.031^{*}$ & $60^{*}$ & $0,19^{*}$ & $1,32 \times 10^{-5^{*}}$ & $2,73^{*}$ \\
\hline Diuron & $233,1^{*}$ & $1,1 \times 10^{-3 *}$ & $36,4^{*}$ & $400^{*}$ & $2,85^{*}$ & $7,04 \times 10^{-6^{*}}$ & - \\
\hline Fluazifop-p-butil & $383,4^{*}$ & $0,033^{*}$ & $1,1^{*}$ & $5.800^{*}$ & $4,5^{*}$ & $1,1 \times 10^{-2^{*}}$ & $-3,1^{*}$ \\
\hline Flumioxazin & $354,3^{*}$ & $0,32^{*}$ & $0,00179^{*}$ & $0,21^{* *}$ & $2,82^{* *}$ & $6,33 \times 10^{-5 * *}$ & - \\
\hline MSMA & $140^{*}$ & $1 \times 10^{-2^{*}}$ & $1,4^{*}$ & 2850 & $<0$ & $1,16 \times 10^{-4 * *}$ & $9,02^{*}$ \\
\hline Oxyfluorfen & $361,7^{*}$ & $0,0267^{*}$ & $0,116^{*}$ & 17.636 & $4,47^{*}$ & $8,32 \times 10^{-2 * *}$ & - \\
\hline Paraquat & $257,2^{*}$ & $<1 \times 10^{-2^{*}}$ & $0,620^{*}$ & $1.000^{* *}$ & $-4,5^{*}$ & $<4 \times 10^{-9^{*}}$ & - \\
\hline Simazina & $201,7^{*}$ & $2,94 \times 10^{-3^{*}}$ & $6,2^{*}$ & $190^{*}$ & $2,1^{*}$ & $5,6 \times 10^{-5^{*}}$ & $1,62^{*}$ \\
\hline Thiadizuron & $220,2^{*}$ & $4 \times 10^{-6^{*}}$ & $31^{*}$ & $0,05^{* *}$ & $1,77^{*}$ & $2,84 \times 10^{-8^{*}}$ & $8,86^{*}$ \\
\hline Trifluralin & $335,3^{*}$ & 6,18 & $0,184^{*}$ & $22.200^{*}$ & $4,83^{*}$ & $15^{*}$ & - \\
\hline
\end{tabular}

sua capacidade de fugacidade. Esta, por sua vez, é dependente da natureza do compartimento e das características físico-químicas do composto. Assim, os valores foram calculados pelo modelo de fugacidade nível I, em que:

$$
C_{i j}=Z_{i j} f_{i}(8) \text {; assim, } j=1 ; 97 \mathrm{e} i=1 ; 8 \text { : }
$$

$i=\operatorname{ar}(1)$, água (2), planta [raiz (3), caule (4) e folha (5)], solo (6), sedimentos (7) e biota aquática (8) e $j=1 ; 97$ herbicidas.

No ar, a fugacidade de um composto $\left(f_{a}\right)$ é igual à sua pressão de vapor, que, quando expressa em concentração, é dada pela equação:

$$
f_{a}=C_{a} R T
$$

em que $C_{a}$, em $\mathrm{mol} \mathrm{m}^{-3}$, é a concentração do composto no ar, $R=8,314 \mathrm{~Pa} \mathrm{~m}^{3} \mathrm{~mol}^{-1}{ }^{\circ} \mathrm{K}^{-1}$ é a constante universal dos gases e $T$ é a temperatura absoluta em graus Kelvin $(K)$. Como conseqüência, a capacidade de fugacidade a partir do $\operatorname{ar}\left(Z_{a}\right)$ é dada por:

$$
Z_{a}=\frac{1}{R T}
$$

$\mathrm{Na}$ água, a fugacidade de um composto dissolvido é aproximadamente equivalente à sua pressão parcial de vapor, descrita pela lei de Henry, a qual é proporcional à sua concentração, isto é:

$$
\mathrm{f}_{w}=\mathrm{HC}_{w}
$$

em que $\left(f_{w}\right)$ é a fugacidade do composto na água, expressa em unidades de Pascal $\left(P_{a}\right) ;\left(P a \mathrm{~m}^{3} \mathrm{~mol}^{1}\right)$ é a constante de Henry do composto; e $C w$, em mol m $\mathrm{m}^{-3}$, é a concentração na água.

Dessa forma, a capacidade de fugacidade da água $\left(Z_{w}\right)$ é dada por:

$$
Z_{w}=\frac{1}{H}
$$

Para outros compartimentos, a fugacidade não possui relação direta com parâmetros físicos do composto; portanto, a capacidade de fugacidade depende do conhecimento da capacidade do composto na água ou no ar (Mackay, 1991). Por exemplo, para solo, sedimento, caule, folha e raiz, a capacidade de fugacidade pode ser estimada pelas expressões de Calamari et al. (1987).

Nessas expressões, a capacidade de fugacidade do solo $\left(Z_{s}\right)$ é dada por:

$$
Z_{s}=0,2 Z_{a}+0,3 Z_{w}+0,5 f_{o c} \rho_{s} K_{o c} Z_{w}
$$

em que $f_{\text {oc }}$ é a fração volumétrica de carbono orgânico do solo; $\rho_{s}$, a densidade do solo, expressa em $\mathrm{g} \mathrm{cm}^{-3} ; K_{o c}$, o coeficiente de partição do 
composto entre o carbono orgânico e a solução do solo. Os valores $0,2,0,3$ e 0,5 representam, respectivamente, o conteúdo volumétrico médio de ar, água e matéria sólida do solo.

A capacidade de fugacidade do sedimento, $Z_{\text {sd }}$, é dada por:

$$
Z_{\text {sd }}=0,5 Z_{w}+0,5 f_{o d} \rho_{\text {sd }} K_{o c} Z_{w}
$$

em que $\rho_{s d}$ é a densidade do sedimento, expressa em $\mathrm{g} \mathrm{cm}^{-3}$. Os valores 0,5 e 0,5 são, respectivamente, o conteúdo volumétrico médio de água e matéria sólida do sedimento.

A capacidade de fugacidade da planta é estimada dividindo-a nos subcompartimentos folha, caule e raiz. Na folha, a capacidade de fugacidade $\left(Z_{1}\right)$ pode ser estimada pela expressão:

$$
Z_{l}=\left(0,18 Z_{a}+0,8 Z_{w}+0,02 K_{o w} Z_{w}\right) \rho_{l} / \rho r_{w}
$$

em que $\rho_{l}, \rho_{w}$ e $K_{\text {ow }}$ são, respectivamente, a densidade média das folhas, a densidade da água (expressa em $\mathrm{g} \mathrm{cm}^{-3}$ ) e o coeficiente de partição do composto entre n-octanol e a água. Os valores $0,18,0,8$ e 0,02 representam, respectivamente, o conteúdo volumétrico médio de ar, água e lipídeos da folha.

No caule, a capacidade de fugacidade $\left(Z_{s t}\right)$ é dada por:

$$
Z_{s t}=\left(0,18 Z_{w}+0,02 K_{o w} Z_{w}\right) \rho_{s t} / \rho_{w}
$$

em que $\rho_{s t}$ é a densidade do caule, expressa em $\mathrm{g} \mathrm{cm}^{-3}$. Os valores 0,82 e 0,02 representam, respectivamente, o conteúdo volumétrico médio de água e lipídeos do caule. A capacidade de fugacidade da raiz $\left(Z_{r}\right)$ é estimada pela relação:

$$
Z_{r}=\left(0,82 Z_{w}+0,014 \mathrm{~K}_{o w} Z_{w}\right) \rho_{r} / \rho_{w}
$$

em que $\rho_{r}$, é a densidade da raiz (expressa em $\mathrm{g} \mathrm{cm}^{-3}$ ). Os valores 0,82 e 0,014 representam, respectivamente, o conteúdo volumétrico médio de água e lipídeos da raiz. Conseqüentemente, a capacidade de fugacidade total da planta é estimada por:

$$
\mathrm{Zp}=\mathrm{Zr}+\mathrm{Zst}+\mathrm{Zl}
$$

Para os compartimentos de natureza biótica correspondentes a organismos residentes na água, como o zooplâncton e o fitoplâncton, a capacidade de fugacidade $\left(Z_{b}\right)$ de um composto, nesses compartimentos, pode ser calculada por:

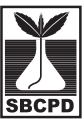

$$
\begin{aligned}
& Z b=Z w B C F \\
& B C F=B C F_{n}(1-\rho)+\rho B C F_{a} \\
& p=\frac{10^{(p h-p k a)}}{1+10^{(p h-p k a)}}
\end{aligned}
$$

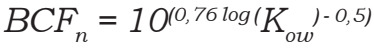

$$
\begin{aligned}
& B C F_{a}=10^{\left(0,76 \log \left(K_{\text {ow }}\right)^{-3,4)-0,5)}\right.}
\end{aligned}
$$

em que $B C F$ é o fator de bioconcentração do composto no organismo aquático.

O modelo de fugacidade nível I foi concebido para um estado em que todas as fugacidades são iguais e constantes em todos os compartimentos; as capacidades de fugacidade, $Z$, são conhecidas e os processos de transferências e degradações são idealmente inexistentes. Nessas condições, mediante um conjunto de fórmulas matemáticas e conhecendo todos os termos/parâmetros/dados dos herbicidas, podem-se calcular as concentrações percentuais do composto em cada um dos compartimentos ou determinar a distribuição percentual da massa total do composto. Assim, se cada compartimento $i$ tem um volume bem definido $\left(V_{i}\right)$, a concentração em cada compartimento $\left(C_{i}\right)$ é igual a $f_{i} Z_{i}=f Z_{i}(24)$, em que $f$ é a fugacidade predominante de equilibrio. A quantidade do composto no compartimento $i, Q$, expressa em mol, é:

$$
Q_{i}=C_{i} V_{i}=f_{i} Z_{i} V_{i}
$$

A quantidade total do composto no sistema ambiental padrão é calculada por:

$$
Q_{T}=f \sum_{i=1}^{n} Z_{i} V_{i} \cdot
$$

Supondo que a quantidade total $\left(Q_{T}\right)$ seja conhecida, a fugacidade pode ser calculada por:

$$
f=\frac{Q_{T}}{\sum_{i=1}^{n} Z_{i} V_{i}}
$$

Em conseqüência, os valores individuais de $C_{i}$ e $Q_{i}$ podem ser estimados por $C_{i}=f Z_{i}(28)$ e $Q_{i}=C_{i} V_{i}(29)$, respectivamente. O percentual de distribuição da massa total do composto no compartimento $i, P_{i}$, pode ser calculado pela equação:

$$
P_{i}=\frac{Z_{i} V_{i}}{\sum_{i=1}^{n} Z_{i} V_{i}} 100 \%
$$


em que $n$ é o número de compartimentos. Dessa forma, os valores de $\rho_{1}$ revelam o compartimento onde se encontra a maior porcentagem do composto químico ou onde o compartimento tem maior solubilidade para o composto.

\section{RESULTADOS E DISCUSSÃO}

Os principais herbicidas utilizados na cultura do algodão - e objeto de análise deste trabalho - foram carfentrazone-ethyl, thiadizuron, trifluralin, clomazone, clethodim, fluzifopp-butil e MSMA. A análise dos resultados mostrou que o herbicida carfentrazone apresentou a tendência de se distribuir pelos compartimentos água $(76,14 \%)$, raiz $(10 \%)$, caule $(9,03 \%)$, solo $(3,19)$ e sedimento $(1,22 \%)$; o clomazone apresentou os mesmos compartimentos na distribuição que o carfentrazone, mas a ordem foi: água $(88,43 \%)$, solo $(5,12 \%)$, raiz $(2,24 \%)$, caule $(2,04 \%)$ e sedimento $(1,95 \%)$. Resultados semelhantes aos deste trabalho foram encontrados por Plese et al. (2006a) e Silva et al. (2007) para o clomazone, os quais realizaram o cálculo do nível I de fugacidade em área de cultivo de arroz e de cana-de-açúcar, respectivamente.

Para MSMA, o compartimento mais vulnerável foi água, cujo valor apresentou $82,10 \%$; solo e sedimento vieram em seguida, com valores na ordem de 12,88 e 4,98\%, respectivamente, inferiores aos do primeiro compartimento. Já clethodim e thidiazuron apresentaram o compartimento água como o mais vulnerável, com os valores de 96,82 e 98,66\%, respectivamente (Figura 1). Em menor valor, o compartimento solo apresentou $1,39 \%$ da quantidade do clethodim distribuído pelos diferentes locais.

Os compartimentos mais vulneráveis foram água, caule, raiz e folha, que apresentaram valores de 51,73, 20,75, 22,99 e 3,57\%, respectivamente, na aplicação do fluazifop-pbutil em área de algodão. Esses resultados foram semelhantes aos obtidos por Plese et al. (2006a) em área de cana-de-açúcar. Para trifluralin, os compartimentos vulneráveis foram raiz e caule, com 51,69 e 46,63\%, respectivamente.

O herbicida simazine, que é aplicado em área de café, apresentou como compartimento mais vulnerável água $(95,06 \%)$, seguido do solo
$(2,80 \%)$, em menor quantidade. A ametrina também demonstrou ter tendência pelo compartimento água $(92,47 \%) \mathrm{e}, \mathrm{em}$ menores valores, por solo $(2,94 \%)$, raiz $(1,75 \%)$, caule $(1,59 \%)$ e sedimento $(1,13 \%)$. Plese et al. (2006b) calcularam o nivel 1 de fugacidade para a simazine e encontraram maior vulnerabilidade para o compartimento água.

O cálculo da concentração em porcentagem, utilizando o nivel I de fugacidade, para o acetochlor, mostrou ter este herbicida principal tendência para os compartimentos raiz $(38,62 \%)$ e caule $(34,84 \%)$. Os compartimentos água, ar e solo apresentaram os valores de 19,77, 3,31 e 2,05\%, respectivamente. Esses resultados corroboram os obtidos por Plese et al. (2006b).

2,4-D, paraquat e diuron são alguns dos principais herbicidas utilizados no controle de plantas daninhas na cultura do algodão, café e citros. O 2,4-D mostrou preferência pelo compartimento água (98,79\%) (Figura 1), enquanto o paraquat tendeu a se deslocar tanto para o solo $(71,41 \%)$ quanto, em menor valor, para o sedimento (27,03\%). Estes resultados estão de acordo com os observados por Plese et al. (2006a, b) e Silva et al. (2007) para o herbicida 2,4-D. Para o diuron, o compartimento água $(87,93 \%)$ apresentou maior vulnerabilidade; em menor valor, seguiram-se solo $(3,67 \%)$, raiz $(3,58 \%)$, caule $(3,24 \%)$ e sedimento $(1,40 \%)$ (Figura 1). Este herbicida é um dos principais produtos aplicados em área de cana-de-açúcar e milho; Plese et al. (2006a, b) observaram a mesma distribuição entre os compartimentos estudados neste trabalho.

As culturas do café e citros apresentam também o oxyfluorfen como um herbicida utilizado no manejo da comunidade infestante, o qual teve uma distribuição similar para os compartimentos raiz $(51,23 \%)$ e caule $(46,21 \%)$ e, em menor quantidade, para o solo, com $1,27 \%$.

Para as áreas de algodão e citros, o herbicida flumioxazin apresentou preferência na distribuição, em ordem decrescente, para os compartimentos água $(93,57 \%)$, solo $(2,13 \%)$, raiz $(1,99 \%)$ e caule $(1,81 \%)$. Os demais compartimentos (ar, biota, sedimento e folha) foram abaixo de 1\% (Figura 1). 

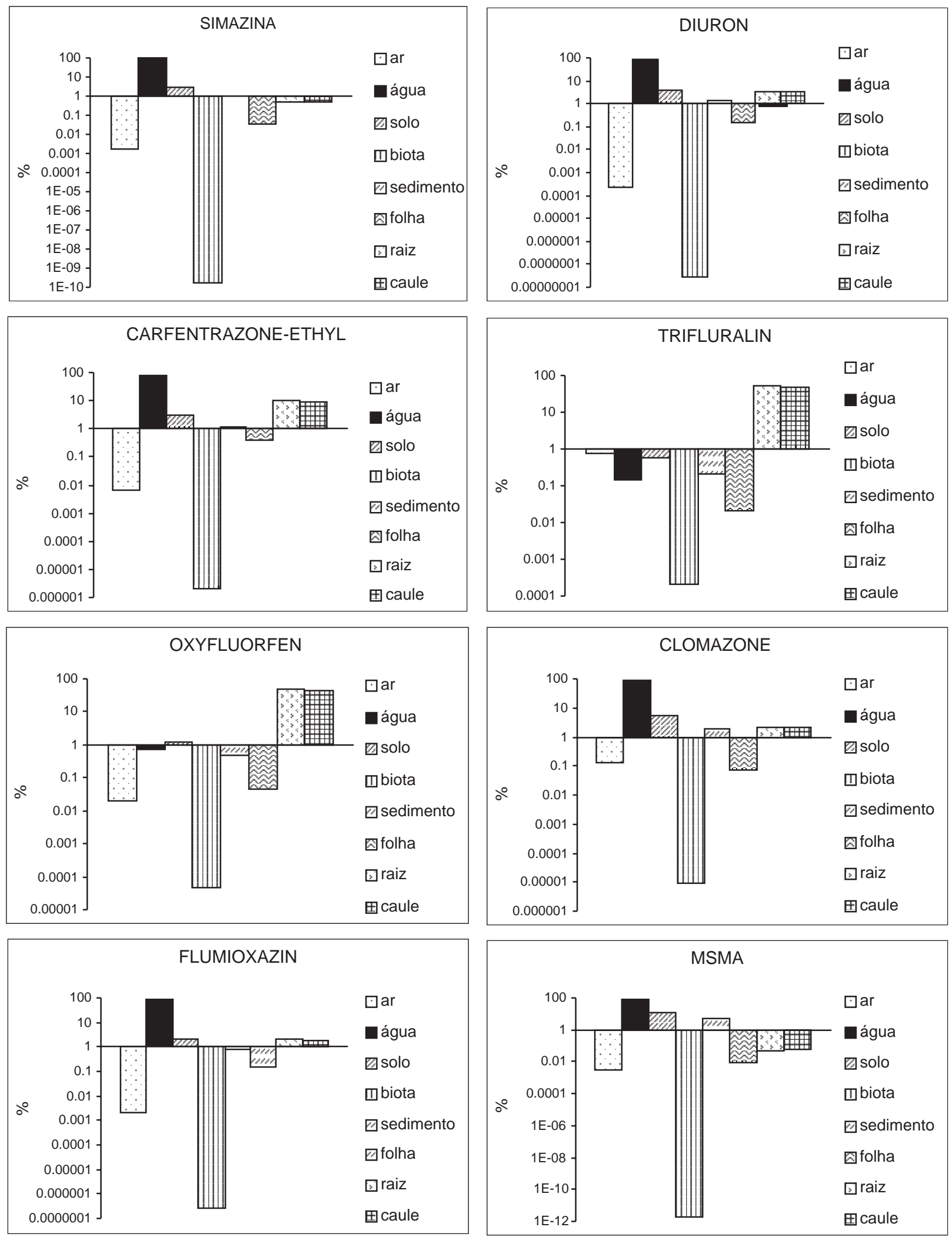

Figura 1 - Diagrama da distribuição dos herbicidas nos compartimentos ambientais em área de algodão, café e citros, aplicando o modelo de fugacidade nível I (continua...) 

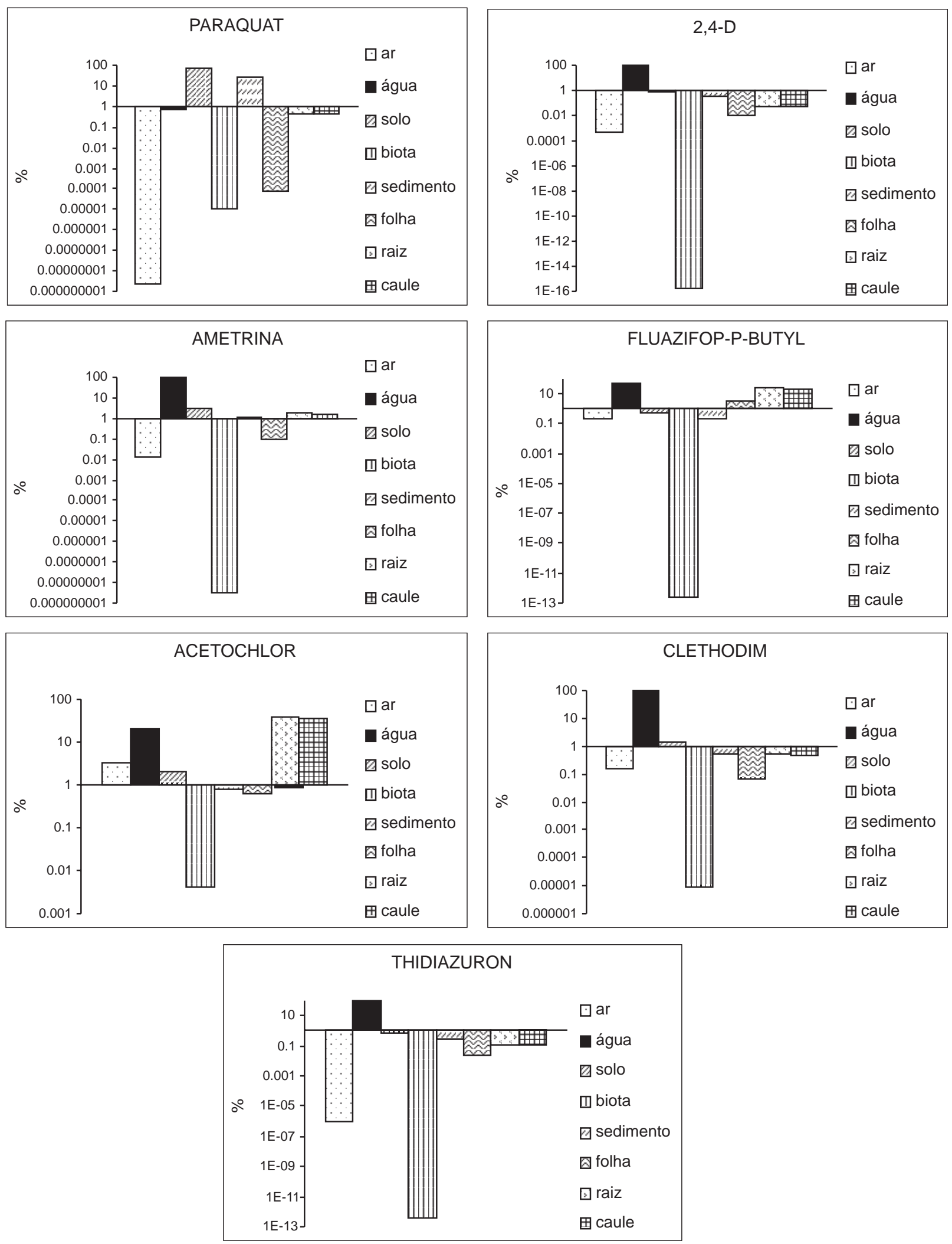

Figura 1 - continuação. 
A fugacidade do acetochlor e trifluralin apresentou valores na ordem de $10^{-6}$ e $10^{-7}$, respectivamente (Tabela 3 ). O paraquat demonstrou ter menor fugacidade na ordem de $10^{-16}$, as dos demais ficaram na ordem de $10^{-8}$ a $10^{-11}$.

As concentrações dos herbicidas nos compartimentos ar, água, solo, biota, sedimento, folha, raiz e caule estão mostradas na Tabela 4.

$\mathrm{Na}$ maioria dos herbicidas avaliados, o compartimento água foi o mais vulnerável. A avaliação da predição utilizando nível de fugacidade (I) demonstrou ser uma ferramenta importante no destino ambiental dos herbicidas estudados para as culturas de algodão, café e citros.
Tabela 3 - Fugacidade dos herbicidas no meio ambiente

\begin{tabular}{|c|c|}
\hline Herbicida & Fugacidade $(\mathrm{Pa})$ \\
\hline Acetochlor & $1,37 \times 10^{-6}$ \\
\hline Ametrina & $5,43 \times 10^{-9}$ \\
\hline Carfentrazone & $2,69 \times 10^{-9}$ \\
\hline Cletodim & $6,31 \times 10^{-8}$ \\
\hline Clomazone & $5,29 \times 10^{-8}$ \\
\hline Diuron & $8,84 \times 10^{-11}$ \\
\hline $2,4-D$ & $1,86 \times 10^{-10}$ \\
\hline Fluazifop-p-butil & $8,13 \times 10^{-8}$ \\
\hline Flumioxazin & $8,42 \times 10^{-10}$ \\
\hline MSMA & $1,36 \times 10^{-9}$ \\
\hline Oxyfluorfen & $8,61 \times 10^{-9}$ \\
\hline Paraquat & $9,21 \times 10^{-16}$ \\
\hline Simazine & $7,60 \times 10^{-10}$ \\
\hline Thidiazuron & $4,00 \times 10^{-13}$ \\
\hline Trifluralin & $3,01 \times 10^{-7}$ \\
\hline & \\
\hline
\end{tabular}

Tabela 4 - Concentração dos herbicidas nos diferentes compartimentos ambientais

\begin{tabular}{|c|c|c|c|c|c|c|c|c|}
\hline \multirow{3}{*}{ Herbicida } & \multicolumn{8}{|c|}{ Compartimento } \\
\hline & $\mathrm{Ar}$ & Água & Solo & Biota & Sedimento & Folha & Raiz & Caule \\
\hline & \multicolumn{8}{|c|}{ Concentração $\left(\mathrm{g} \mathrm{m}^{-3}\right)$ - } \\
\hline Acetochlor & $1,49 \times 10^{-7}$ & 0,000762 & 0,012302 & 0,163442 & 0,009576 & 0,175117 & 2,816631 & 2,043947 \\
\hline Ametrina & $6,51 \times 10^{-10}$ & 0,003938 & 0,019493 & $1,33 \times 10^{-7}$ & 0,015358 & 0,031006 & 0,141114 & 0,103280 \\
\hline Carfentrazone & $4,47 \times 10^{-10}$ & 0,004484 & 0,029251 & 0,000125 & 0,022950 & 0,173911 & 1,114868 & 0,809968 \\
\hline Cletodim & $9,16 \times 10^{-9}$ & 0,004978 & 0,011193 & 0,000470 & 0,009002 & 0,024704 & 0,050671 & 0,037887 \\
\hline Clomazone & $5,11 \times 10^{-9}$ & 0,003028 & 0,027287 & 0,000319 & 0,021331 & 0,018302 & 0,145573 & 0,106311 \\
\hline 2,4-D & $2,24 \times 10^{-11}$ & 0,004207 & 0,005174 & $7,79 \times 10^{-15}$ & 0,004291 & 0,003515 & 0,003633 & 0,003583 \\
\hline Diuron & $8,32 \times 10^{-12}$ & 0,002928 & 0,019034 & $9,55 \times 10^{-7}$ & 0,014935 & 0,036720 & 0,225854 & 0,164541 \\
\hline Fluazifop-p-butil & $9,78 \times 10^{-9}$ & 0,002203 & 0,003530 & $1,09 \times 10^{-11}$ & 0,002881 & 1,157138 & 1,852719 & 1,344847 \\
\hline Flumioxazin & $1,2 \times 10^{-10}$ & 0,004711 & 0,016773 & $1,31 \times 10^{-5}$ & 0,013305 & 0,056452 & 0,191435 & 0,139967 \\
\hline MSMA & $1,63 \times 10^{-10}$ & 0,003497 & 0,085327 & $7,41 \times 10^{-11}$ & 0,066296 & 0,002843 & 0,003991 & 0,003683 \\
\hline Oxyfluorfen & $1,26 \times 10^{-9}$ & $3,74 \times 10^{-5}$ & 0,010234 & 0,002458 & 0,007926 & 0,018338 & 5,008486 & 3,634215 \\
\hline Paraquat & $1,10 \times 10^{-16}$ & $3,05 \times 10^{-5}$ & 0,473102 & 0,000454 & 0,366274 & $2,67 \times 10^{-5}$ & 0,035328 & 0,025641 \\
\hline Simazine & $9,14 \times 10^{-11}$ & 0,004048 & 0,018596 & $7,37 \times 10^{-9}$ & 0,014671 & 0,011690 & 0,042141 & 0,031489 \\
\hline Thidiazuron & $4,82 \times 10^{-14}$ & 0,004202 & 0,005157 & $1,52 \times 10^{-11}$ & 0,004277 & 0,007464 & 0,008481 & 0,007100 \\
\hline Trifluralin & $4,07 \times 10^{-8}$ & $6,73 \times 10^{-6}$ & 0,004178 & 0,009982 & 0,003235 & 0,007558 & 4,684975 & 3,399465 \\
\hline
\end{tabular}

\section{LITERATURA CITADA}

BRU, R.; CARRASCO, J. M.; PARAÍBA, L. C. Unsteady state fugacity model by a dynamic control system. Appl. Math Model., v. 22, n. 7, p. 485-494, 1998.

CALAMARI, D.; VIGHI, M.; BACCI, E. The use of terrestrial plant biomass as a parameter in the fugacity model. Chemosphere, v. 16, n. 10-12, p. 2359-2364, 1987.
MACKAY, D.; PATERSON, S. Calculating fugacity. Environ. Sci. Technol., v. 15, n. 9, p. 1006-1014, 1981.

MACKAY, D. Multimedia environmental models: the fugacity approach. Boca Raton: Lewis Publishers, 1991. $257 \mathrm{p}$.

PARAÍBA, L. C. Modelización de la fugacidad mediante sistemas dinámicos discretos. 1999. 199 f.. Tesis (Doctoral) - Universidad Politécnica de Valência, Valência, 1999. 
PARAÍBA, L. C.; CARRASCO, J. M.; BRU, R. Level IV fugacity model by continuous time control system. Chemosphere, v. 38, n. 8, p. 1763-1775, 1999.

PARAÍBA, L. C. et al. Simulation of the fate of the insecticide carbifuran in a rice field using a level IV fugacity model. Span J Agric Res., v. 5, n. 1, p. 43-50, 2007.

PLESE, L. P. M.; SILVA, C. L.; FOLONI, L. L. Previsão da distribuição ambiental dos herbicidas utilizados na cultura da cana-de-açúcar. CONGRESSO BRASILEIRO DA CIÊNCIA DAS PLANTAS DANINHAS, 25., 2006. Brasília. Anais... Brasília: 2006a. p. 133.
PLESE, L. P. M.; SILVA, C. L.; FOLONI, L. L. Previsão da distribuição ambiental dos herbicidas utilizados na cultura do milho. CONGRESSO BRASILEIRO DA CIÊNCIA DAS PLANTAS DANINHAS, 25., 2006, Brasília. Anais... Brasília: 2006a. p. 134.

SILVA, C. L. et al. Previsão ambiental da distribuição dos pesticidas aplicados na cultura do arroz. Pestic.: $\mathbf{R}$. Ecotoxicol Meio Amb., v. 17, n. 1, p. 75-86, 2007.

TOMLIN, C. D. S. The pesticide manual. Farnham: British Crop Protection Council, 2000.

YENIGÜN, O.; SOHTORIK, D. Calculation with the level II fugacity model for selected organophosphorus insecticides. Water Air Soil Poll., v. 84, n. 1, p. 175-185, 1995. 